\title{
Carnívoros del Área de Protección de Flora y Fauna Sierra de Quilla, Jalisco
}

\author{
María Magdalena Ramírez-Martínez ${ }^{*}$, \\ Luís Ignacio Iñiguez-Dávalos ${ }^{1}$ y Martha Pilar Ibarra-López ${ }^{1}$
}

Introduction. A central strategy for conservation in México is the creation of natural protected areas. Their management requires providing adequate information on wildlife to guide the management decision process. Within the 17 protected areas that exist in Jalisco, mammal knowledge is scarce, except for three of the most studied areas in the state: Manantlán, Chamela-Cuixmala, and Primavera forest. Because of their taxonomic and ecological diversity, carnivores are an important group to evaluate protected areas and management and conservation actions. Our aim is to provide basic information on carnivores of the Sierra de Quila Flora and Fauna Protection Area, in the State of Jalisco.

Method. The Sierra de Quila is part of the Physiographic Province Neo-volcanic Axis and the sub-province Sierras de Jalisco located in the central part of the state and bordered to the north by the Sierra Madre Occidental, on the northwest by the Mesa Central, and west and south by the Sierra Madre del Sur. A total of 10 field trips were performed from November 2008 to February 2010, lasting three days each. Five vegetation types were sampled using both direct and indirect methods: track searching, scent stations, and trap-cameras.

Results. Of the 16 potential carnivore species for the area, 13 species of this order were recorded $(81 \%$ of potential species, Table 2). The other three carnivores reported for the area were included, but they were not recorded in our study. The richest family was Felidae with four species; two of them are classified as endangered. Depending on the sampling method, gray fox (tracks), coyote (scent stations), or puma (trapcameras) was the most abundant carnivore in the area. Activity patterns shown that four species were mainly nocturnal; two of them were diurnal and two were cathemeral (day / night; Figure 2).

Discussion and Conclusion. Despite the deterioration shown in some vegetation types within the area, the presence of a number of species of carnivores gives us an idea of ecosystem resilience where even species with the priority to establish conservation programs are found as the case of two cat species recorded with endangered category (P) according to NOM-ECOL-059-2010 (DOF 2010). It is necessary to continue inventory work on carnivores of this protected area, especially in the lower part of the altitudinal range and evaluate the impact of human activities in their presence and abundance.

Key words: Biodiversity, carnivores, conservation, Jalisco, natural protected areas

\section{Resumen}

Una estrategia central de conservación en México es el establecimiento de áreas protegidas. El manejo adecuado de éstas requiere un conocimiento suficiente de su biota, para orientar la toma de decisiones. Por su diversidad taxonómica y ecológica, los mamíferos carnívoros constituyen un grupo importante para evaluar las áreas protegidas y dar seguimiento a las acciones de conservación y manejo. Este trabajo busca aportar información básica de los carnívoros para el Área de Protección de Flora y Fauna Sierra de Quila, en el Estado de Jalisco.

\footnotetext{
${ }^{1}$ Departamento de Ecología y Recursos Naturales, Centro Universitario de la Costa Sur, Universidad de Guadalajara. Av. Independencia Nacional 151, Autlán 48900, Jalisco, México. E-mail: maleni.ramirezm@gmail.com (MMR-M)

* Corresponding author
} 
La Sierra de Quila forma parte de la Provincia Fisiográfica Eje Neovolcánico y de la Subprovincia Sierras de Jalisco (Guerrero y Cházaro, 1995), que se localiza en la parte central del estado y limita al norte con la Sierra Madre Occidental, al noroeste con la Mesa del Centro y al oeste y sur con la Sierra Madre del Sur. Se realizaron 10 salidas de campo entre noviembre de 2008 y febrero de 2010, con una duración de tres días cada una. Se muestrearon cinco tipos de vegetación, utilizando métodos directos e indirectos, como búsqueda de rastros, cámaras trampa y estaciones olfativas.

De las 16 especies potenciales de carnívoros para el área (Iñiguez-Dávalos y Santana-C., 2004), se han registrado 13 especies de este orden $(81 \%$ de las especies potenciales; Tabla 2). Se incluyen tres carnívoros reportados para el área pero no registrados en nuestro estudio. Destaca la familia Felidae, con cuatro especies, dos de las cuales están catalogadas como en peligro de extinción. Dependiendo del tipo de muestreo utilizado se destacó la zorra gris (rastros), el coyote (estaciones olfativas) y el puma (cámaras trampa) como la especie más abundante del área. Los patrones de actividad mostraron que cuatro especies son nocturnas, dos fueron diurnas y dos catemerales (diurna/nocturna; Figura 2).

A pesar del deterioro que muestran algunos tipos de vegetación dentro del área, la presencia de un buen número de especies de carnívoros nos da una idea de la resiliencia del ecosistema, en el que incluso podemos encontrar especies que son prioritarias para establecer programas de conservación, ya que se tienen el registro de dos especies de felinos con categoría en peligro de extinción (P) según la NOM-ECOL-059-2010 (DOF 2010). Es necesario continuar con el inventario de carnívoros, sobre todo en la parte baja del intervalo altitudinal, la selva baja y partes bajas de los ríos así como evaluar el impacto de las actividades antrópicas en su presencia y abundancia.

Palabras clave: Áreas naturales protegidas, biodiversidad, carnívoros, conservación, Jalisco

Los mamíferos carnívoros constituyen uno de los grupos más interesantes para evaluar las áreas naturales protegidas y dar seguimiento a las acciones de conservación y manejo que se realizan en estas. Su papel como reguladores de poblaciones de las presas modela la estructura de la comunidad tanto de los mismos animales, como indirectamente de las plantas mediante efectos de cascadas tróficas (Terborgh et al. 2010). Por otro lado, la necesidad de ámbitos hogareños generalmente amplios y con buena calidad que tiene un buen número de ellos los convierte tanto en especies sombrilla para la conservación, como en indicadores de la calidad del ecosistema (Caro y O’Doherty 1999; Bond 2010).

Esto permite, mediante el análisis de la comunidad y sus poblaciones, valorar diversos aspectos de la integridad del ecosistema, como podrían ser el grado de conservación del hábitat, la presencia de factores de perturbación antrópicos (fuego, ganado, cazadores, entre otros) y naturales, la disponibilidad de diversos recursos de refugio, agua y alimento (incluyendo, en la mayoría de los casos, las poblaciones de otros animales presa) y sus relaciones interespecíficas, que pueden llevar tanto a la competencia como a la especialización en el uso de los recursos (Spowart y Samson 1986). 
El occidente de México (tradicionalmente considerado como los estados de Nayarit, Jalisco, Colima, Michoacán y Guanajuato) es una región que se caracteriza por ser una zona de transición y de una gran diversidad geográfica y biológica (Ramamoorty et al. 1998). La complejidad del relieve, la litología y la historia geológica, así como su ubicación en una zona de transición biogeográfica entre las regiones Neotropical y Neártica, son el marco de una alta diversidad de ecosistemas donde se pueden encontrar desde sistemas estuarinos, bosques tropicales (caducifolios y subcaducifolios), y matorrales, hasta bosques subtropicales y templados (Jardel et al. 1996; Fa y Morales 1998).

En consecuencia, la región sostiene una rica diversidad de flora y fauna; tan solo en Jalisco se encuentran 7,000 especies de plantas vasculares, existen registros de 525 especies de aves, y en la costa de Jalisco se han reportado 66 especies de reptiles y 19 de anfibios, 42 de las cuáles son endémicas a México (García y Ceballos 1994; Jardel et al. 1996). En general, la mayoría de los endemismos de México, tanto de mamíferos y los demás vertebrados, así como de invertebrados y plantas vasculares, están en el occidente del país, concentrados principalmente en la Sierra Madre Occidental, el Eje Neovolcánico Transversal, la parte norte de la Sierra Madre del Sur y la Cuenca del Río Balsas (Iñiguez Dávalos y Santana-C 1993; Flores Villela y Geréz 1994; Escalante Pliego et al. 1998; Fa y Morales 1998; Flores Villela 1998).

En cuanto a mamíferos, en el Estado de Jalisco se han registrado 172 especies terrestres ubicadas dentro de 23 familias y nueve órdenes. Esto representa el $38.2 \%$ de la mastofauna del país, con un nivel de endemismo del $8.2 \%$ con respecto a México y 21.5\% con respecto al Estado de Jalisco (Iñiguez Dávalos y Santana-C 2004). El orden Carnívora representa el tercer lugar en cuanto a la riqueza de especies del estado, con 20 especies; sin embargo, vale la pena destacar que es el grupo con más especies en alguna categoría de conservación según la NOM-059-2010, con siete especies (Iñiguez Dávalos y Santana-C 2004; DOF 2010). Por lo tanto, es relevante revisar algunas de las estrategias de conservación que se han implementado para su protección.

Una de las estrategias centrales en la política de conservación en México y el mundo es el establecimiento de áreas naturales protegidas. El adecuado manejo de un área protegida requiere un conocimiento adecuado de su biota, para orientar la toma de decisiones, generalmente siguiendo un esquema de manejo adaptativo (Feisinger 2001). En las 17 áreas naturales protegidas que se han decretado en el Estado de Jalisco, hay un conocimiento muy superficial de su mastofauna, y solo en tres de ellas se ha comenzado a profundizar su estudio: Manantlán, Chamela-Cuixmala y Bosque La Primavera. Este trabajo tiene como objetivo proporcionar un aporte al inventario de los mamíferos carnívoros para el Área de Protección de Flora y Fauna Sierra de Quila, dado que este listado no ha sido compilado hasta ahora y como tal, constituirá un aporte al plan de manejo que se encuentra en proceso de elaboración. Así mismo, se quiere hacer una primera aproximación para evaluar la importancia relativa de las especies dentro del ensamblaje de carnívoros del área protegida. Finalmente, se busca describir los patrones de actividad de los carnívoros más abundantes del área. 


\section{Material y Métodos}

Área de estudio. El Área de Protección de Flora y Fauna Sierra de Quila (APFFSQ) fue decretada el cuatro de agosto de 1982. Tiene una extensión de 15,192 ha, y se ubica a $120 \mathrm{~km}$ de la ciudad de Guadalajara (Fig. 1). El polígono del área natural protegida se sitúa entre las coordenadas geográficas extremas $20^{\circ} 14.650$ y $20^{\circ} 21.707$ de latitud Norte y $-103^{\circ} 57.288$ y $-104^{\circ} 7.590$ de longitud Oeste, dentro de los municipios de San Martín Hidalgo, Tecolotlán y Tenamaxtlán en el Estado de Jalisco, México. La Sierra de Quila forma parte de la Provincia Fisiográfica Eje Neovolcánico y de la Subprovincia Sierras de Jalisco (Guerrero y Cházaro, 1995), que se localiza en la parte central del estado y limita al norte con la Sierra Madre Occidental, al noroeste con la Mesa del Centro y al oeste y sur con la Sierra Madre del Sur. De manera general su relieve es montañoso con valles de poca extensión; la topografía es muy accidentada, con pendientes del orden de $15^{\circ}$ a $60^{\circ}$ (Guerrero y Cházaro 1995). El APFFSQ presenta un rango altitudinal que va de los 1,350 m en su zona más baja, a los 2,560 m. Su clima es templado subhúmedo con Iluvias en verano, en las partes más altas, mientras que en las partes bajas es templado semicálido; la precipitación media anual es de 900 mm (García 1988; Villavicencio et al. 2005).

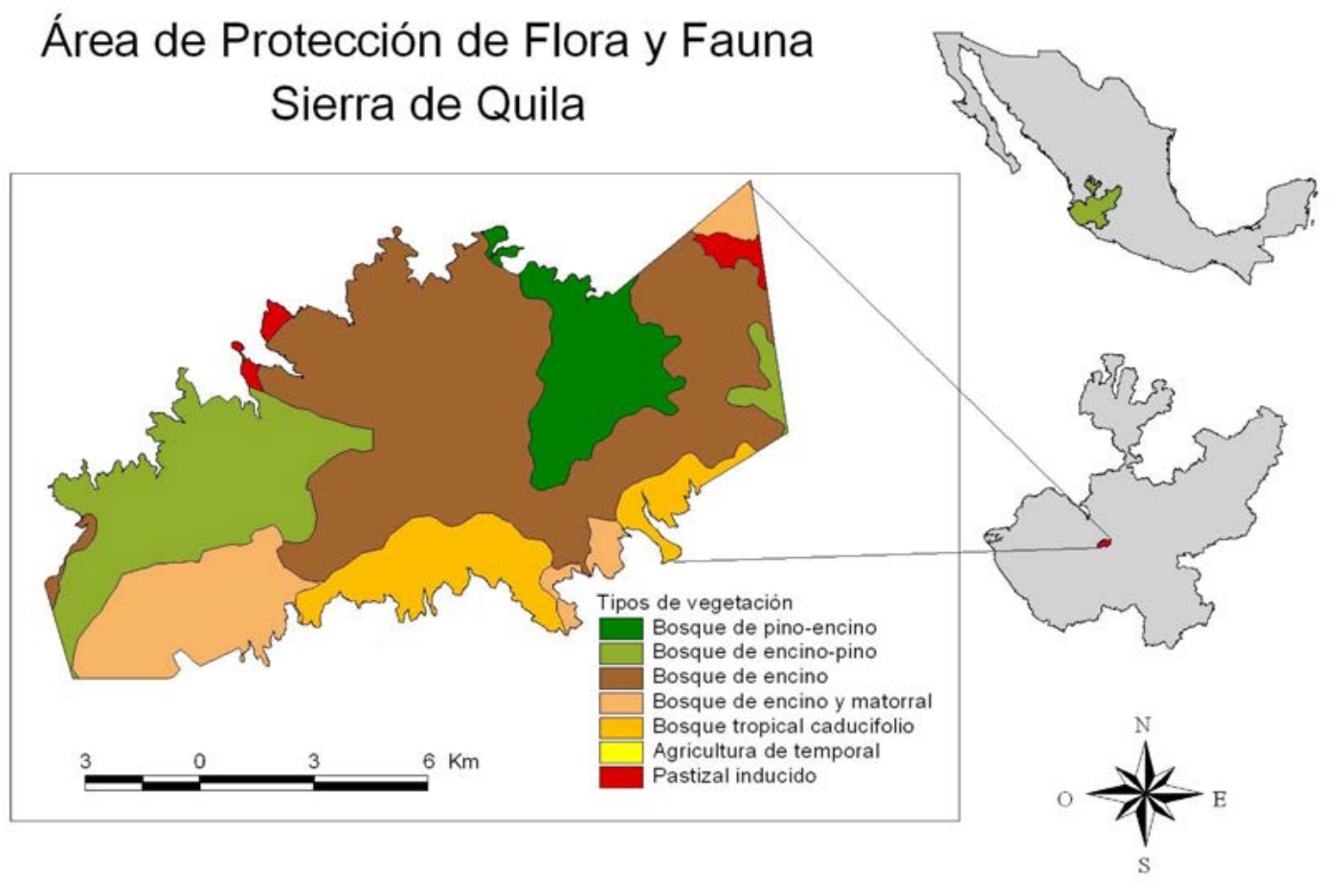

Figura 1. Localización del Área de Protección de Flora y Fauna Sierra de Quila. Las unidades de las coordenadas están en metros, pues el sistema de proyección del mapa es UTM.

El Área de Protección de Flora y Fauna Sierra de Quila capta agua para dos cuencas hidrológicas de Jalisco; la porción norte drena hacia el río Ameca y la ladera sur alimenta el río Ayuquila-Armería (CEA 2011). Es una zona de gran importancia para la recarga de acuíferos en la región centro del Estado de Jalisco (SEMADES 2010). La diversidad de condiciones geográficas, edáficas y climáticas de la sierra ha resultado en la presencia de seis tipos de vegetación: bosque de pino-encino (56.5\% de cobertura en el ANP), bosque de encino-pino (5.9\%), bosque de encino (16.9\%), bosque tropical caducifolio 
(14.3\%), terrenos cubiertos por pastizal (2.8\%) y la vegetación de las barrancas y áreas riparias, que consta de pequeños relictos de bosque mesófilo de montaña y bosques de galería (Villavicencio et al. 2005).

Trabajo de campo. Se realizaron 10 salidas de campo entre noviembre de 2008 y febrero de 2010, con una duración de tres días cada una. Se utilizaron diferentes métodos directos e indirectos (búsqueda de rastros, uso de cámaras trampa y estaciones olfativas), que son complementarios, para tener más oportunidad de registrar a la mayoría de las especies. Para ello se realizó un reconocimiento del área utilizando sistemas de información geográfica y luego haciendo un recorrido de campo, que permitió identificar sitios adecuados para el desarrollo de los muestreos. Se ubicaron caminos y veredas con diferente grado de accesibilidad y suficientemente limpias para la colocación de trampas, así como con substrato suave que permitiera la fácil identificación de los rastros en las estaciones olfativas. Todos los registros fueron georeferenciados con un GPS.

Nuestro esfuerzo de captura abarcó cinco tipos de vegetación, aunque no con la misma intensidad (Tabla 1); solo en el pastizal no fue posible realizar muestreos debido a que la superficie cubierta por este tipo de vegetación es muy pequeña y las trampas quedaban muy visibles, lo que aumenta el riesgo de que sean removidas del sitio. Aunque el rango altitudinal dentro del polígono bajo decreto va de 1,350 a 2,560 m, el esfuerzo de muestreo estuvo centrado en las cotas altitudinales que están entre los 1,990 y $2,476 \mathrm{~m}$.

Tabla 1. Distribución de las unidades de muestreo por tipo de vegetación en el Área de Protección de Flora y Fauna Sierra de Quila.

\begin{tabular}{|lccc|}
\hline \multicolumn{5}{c}{ Tipo de muestreo } \\
\hline Tipo de vegetación & Colecta de excretas & Estaciones olfativas & Cámaras-trampa \\
\hline Pino-encino & 43 & 40 & 12 \\
Encino & 4 & 5 & 17 \\
Encino-pino & 6 & 13 & 6 \\
Tropical caducifolio & 1 & 5 & 0 \\
Barrancas & 0 & 0 & 11 \\
\hline
\end{tabular}

Método para búsqueda de rastros. Una vez localizados los caminos y veredas adecuados, se realizaron ocho transectos de $2.5 \mathrm{~km}$ de largo para localizar rastros de mamíferos. Se trató de abarcar la mayor extensión posible, cubriendo los diferentes tipos de vegetación presentes en el área. Estos transectos se recorrieron a pie en cada muestreo, haciendo un registro sistemático de los rastros encontrados e identificando la especie a la cual pertenecen; los recorridos se hicieron durante la mañana, por cuatro horas, con dos personas cada uno. Las excretas fueron fotografiadas y colectadas y, en función de sus características, asignadas a una especie utilizando las guías de campo de Aranda (2000, 2012). Para el caso de los registros de huellas, se fotografiaron y se procuró realizar al menos una impresión para cada especie, sobre todo cuando éstas representaban cierta dificultad para su identificación. Como esfuerzo de colecta se utilizó la sumatoria del total de kilómetros recorridos por persona durante todo el muestreo. 
Método de estaciones olfativas. Se colocaron 63 estaciones olfativas en ocho transectos que se diseñaron para ser de 3,600 m, con 10 estaciones separadas $400 \mathrm{~m}$ una de la otra. Sin embargo, la longitud del transecto varió dependiendo del número de estaciones que se colocaron, ya que en algunas ocasiones el terreno o las condiciones climáticas no permitieron poner las 10 estaciones originalmente programadas; todas las estaciones fueron georeferenciadas. Cada estación olfativa consiste en un círculo de tierra suelta y cernida, de aproximadamente un metro de diámetro, en el cual se coloca un cebo dentro de un bote pequeño con orificios, amarrado a una varilla que se entierra en el centro de la estación. El cebo está constituido de vísceras y carne en descomposición, lo cual atrae sobre todo a los animales carnívoros generalistas (Day et al. 1987). Las huellas encontradas en las estaciones se identificaron a nivel de especie, con la ayuda de las claves de identificación de Aranda (2000, 2012).

Método de cámaras-trampa. Se instalaron en total 46 cámaras Wild View 2 Mpx, colocadas en sitios donde el paso de animales es evidente por la frecuencia de rastros encontrados o por la topografía del terreno, como brechas bien habilitadas o riberas de ríos y arroyos permanentes. Las cámaras se colocaron en diferentes sitios tratando de abarcar la mayor cantidad de área posible; el número de cámaras colocadas varió dependiendo de los sitios muestreados y la accesibilidad a los mismos; se programaron para registrar fecha y hora, y se revisaron mensualmente.

Análisis de datos. En principio, la intención del proyecto fue obtener el listado de los carnívoros presentes en el APFFSQ. Sin embargo, los datos obtenidos mediante métodos directos e indirectos pueden dar una idea aproximada sobre la importancia relativa de las especies dentro de la comunidad, aclarando que los métodos utilizados no reflejan la densidad absoluta de cada una de las especies en el área. Para presentar el listado lo más completo posible, a las especies encontradas en el trabajo de campo se agregaron tres especies de carnívoros que no fueron registradas dentro del proyecto; estos fueron reportes documentados previamente mediante registros fotográficos (Valle, com. pers.). Estas tres especies no fueron incluidas en los análisis posteriores.

Abundancia relativa. Por cada método utilizado en este estudio se calculó la frecuencia de ocurrencia para cada una de las especies. En el caso de la colecta de excretas, cada excreta fue considerada como un registro independiente. En las estaciones olfativas, las huellas encontradas en cada estación se consideran como un registro independiente (Aranda 2000). Para las cámaras-trampa se entiende por registro independiente una o más fotografías de un individuo reconocible para cada ciclo de 24 hrs (Yasuda 2004). Cuando en un día se tomaron varias fotos de la misma especie en la misma cámara, se consideró la foto que presentara mayor número de individuos y ese registro fue el considerado para la base de datos de foto-registros independientes. Si en la foto aparecen dos individuos, entonces se consideran dos registros independientes y el número de registros varió según el número de individuos fotografiados (Monroy-Vilchis et al. 2011). Las frecuencias de ocurrencia obtenidas se transformaron en abundancias relativas (Aranda 2000; Monroy-Vilchis et al. 2011): 


$$
\text { Abundancia relativa } \frac{\text { No. de registros por especie }}{\text { No. total de registros }} \cdot 100
$$

Patrones de actividad. Para las especies registradas en las cámaras trampa, se analizó su presencia a lo largo del día. Los datos registrados fueron: especie, fecha y hora de la fotografía. Se determinó el intervalo de tiempo: las horas del día incluyeron el periodo comprendido entre las 6:01 a las 20:00 hrs, y la noche como el periodo entre las de 20:01 y las 6:00 hrs. Las especies se clasificaron como diurnas cuando presentaron más del $90 \%$ de su actividad durante el día, como nocturnas aquellas con más del $90 \%$ de registros durante la noche, y como catemerales aquellas que presentaron más del $10 \%$ de actividad en los dos horarios (Van Schaik y Griffiths 1996; Tattersall 2006).

\section{Resultados}

Colecta de excretas y otros rastros. El esfuerzo de muestreo fue de $20 \mathrm{~km}$ recorridos, sumando todos los transectos. En total se colectaron 83 excretas, de las cuales 54 se pudieron identificar con certeza para cuatro especies: coyote (Canis latrans), zorra gris (Urocyon cinereoargenteus), puma (Puma concolor) y zorrillo de espalda blanca (Conepatus leuconotus) en tres familias de carnívoros (Tabla 2), mientras que al resto no fue posible identificarlas.

Tabla 2. Listado de especies de mamíferos del orden Carnivora encontradas en el Área de Protección de Flora y Fauna Sierra de Quila. Tipos de muestreo: EX, Excretas, $\mathrm{EO}$, Estaciones olfativas, CT, Cámaras-trampa, OTROS, Avistamientos, restos de alimento y sonidos. La categoría corresponde a la lista de especies en riesgo en la NOM-ECOL-059-2010:

P, Peligro de extinción, A, Amenazada;

*, Especies reportadas para el área de estudio, pero no registradas en este trabajo. Para el arreglo taxonómico se siguió el trabajo de Godínez et al. (2011).

\begin{tabular}{|c|c|c|c|c|c|c|}
\hline Familia & Especies registradas & Categoría & EX & EO & $\mathrm{CT}$ & OTROS \\
\hline Felidae & Leopardus pardalis & $\mathbf{P}$ & & & $\bullet$ & \\
\hline Felidae & Leopardus wiedii & $\mathbf{P}$ & & & $\bullet$ & \\
\hline Felidae & Lynx rufus & & & & & $*$ \\
\hline Felidae & Puma concolor & & $\bullet$ & & $\bullet$ & \\
\hline Canidae & Canis latrans & & $\bullet$ & $\bullet$ & $\bullet$ & $\bullet$ \\
\hline Canidae & Urocyon cinereoargenteus & & $\bullet$ & $\bullet$ & $\bullet$ & \\
\hline Mustelidae & Lontra longicaudis & A & & & & $*$ \\
\hline Mustelidae & Mustela frenata & & & $\bullet$ & & \\
\hline Mephitidae & Conepatus leuconotus & & $\bullet$ & $\bullet$ & $\bullet$ & \\
\hline Mephitidae & Mephitis macroura & & & $\bullet$ & & \\
\hline Procyonidae & Bassariscus astutus & & & $\bullet$ & $\bullet$ & \\
\hline Procyonidae & Nasua narica & & & & $\bullet$ & \\
\hline Procyonidae & Procyon lotor & & & & & $*$ \\
\hline
\end{tabular}

Estaciones olfativas. El esfuerzo de muestreo fue de 121 noches/trampa. Se obtuvieron 32 registros independientes, de los cuales 25 correspondieron al orden Carnívora, registrando seis especies: coyote, zorra gris, cacomixtle (Bassariscus astutus), zorrillo de espalda blanca, zorrillo listado (Mephitis macroura) y comadreja (Mustela frenata). Las dos últimas solo se registraron con este método.

Cámaras-trampa. De las 46 cámaras colocadas, 18 de ellas estuvieron de forma semipermanente por periodos prolongados de tiempo (45 días), mientras que el resto (28 cámaras) se utilizaron por una o dos noches en sitios cercanos a las estaciones 
olfativas; el esfuerzo total de muestreo fue de 866 días/trampa. Se obtuvieron un total de 1849 fotografías, de las cuales 425 fueron registros de fauna silvestre y 142 son fotoregistros independientes. Con este método se obtuvo una buena representación del orden Carnívora, con 5 familias y 8 especies (tabla 2): coyote, zorra gris, zorrillo de espalda blanca, cacomixtle, puma, tejón (Nasua narica), ocelote (Leopardus pardalis) y tigrillo (Leopardus wiedii). Estas tres últimas solo fueron registradas con este método.

Riqueza y abundancia relativa. De las 16 especies potenciales de carnívoros para el área (Iñiguez-Dávalos y Santana-C. 2004), se han registrado 13 especies de este orden (81\% de las especies potenciales; Tabla 2). En esta tabla se incluyen tres carnívoros reportados para el área pero no registrados en nuestros datos (ver métodos): mapache (Procyon lotor), lince (Lynx rufus) y nutria (Lontra longicaudis); estas tres especies no se incluirán en análisis subsiguientes. Las 13 especies de carnívoros corresponden a cinco familias, destacando la familia Félidae con cuatro especies, dos de las cuales, el ocelote y el tigrillo, están catalogadas en la NOM-059-2010 como "En peligro de extinción". Le siguen las familias Procyonidae, con tres especies, y Canidae, Mephitidae y Mustélidae con dos especies cada una. Los registros de especies a través de la colecta de excretas mostraron mayor abundancia relativa para la zorra gris $(38.5 \%)$, seguida del coyote (18.1\%), puma (4.8\%), y zorrillos (3.6\%); cabe aclarar que de estos últimos no se pudo llegar a determinar la especie a la que pertenecían. El resto de las excretas colectadas $(35.0 \%)$ no fueron identificadas. La especie con mayor número de registros en las estaciones olfativas fue el coyote $(48.0 \%)$, seguida por el zorrillo de espalda blanca $(20 \%)$, cacomixtle $(16.0 \%)$, zorra gris $(8.0 \%)$, zorrillo rayado y comadreja (4.0 \% cada uno). El método de las cámaras trampa aportó los datos más numerosos; las especies de carnívoros con la mayor cantidad de foto-registros fueron puma (44.7\%), coyote (15.8 $\%)$, zorra gris y tejón (10.5 \% cada uno), cacomixtle (7.9\%), zorrillo de espalda blanca (5.3\%), ocelote y tigrillo (2.6\% cada uno).

Patrones de actividad. De las ocho especies de carnívoros registradas con las cámaras trampa, el coyote (6 foto-registros independientes o FRI), el cacomixtle (3 FRI), el zorrillo espalda rayada (2 FRI) y el tigrillo (1 FRI) son principalmente nocturnas. El tejón (4 FRI) y el ocelote (1 FRI) son principalmente diurnos. El puma presentó $10 \mathrm{FRI}$ nocturnos y 7 diurnos, mientras que la zorra gris tuvo 2 FRI diurnos y 2 FRI nocturnos, lo que las ubica como especies catemerales (Fig. 2).

La Sierra de Quila fue decretada como área protegida hace 32 años; sin embargo, tiene una historia de ocupación desde antes de la conquista, por pueblos indígenas del centro y sur del país, en lo que se conoció como Reino de Chimalhuacán. La ocupación por parte de los españoles data de 1525 (Agraz 1950). Por tanto, sabemos que desde hace más de 500 años el área ha sufrido una fuerte presión por parte de la población humana, con las modificaciones a los hábitats que esto implica. En esta área se desarrollan actividades productivas como agricultura, ganadería y apicultura y aprovechamiento de recursos naturales silvestres para su venta (hongos, madera muerta, tierra de monte, colecta de frutos, carbón). Así mismo, ha sido sometida a presiones y perturbaciones 
debido a aprovechamientos forestales maderables de manera legal e ilegal; presenta en su historial fuertes incendios forestales y cambio de uso de suelo para agricultura y agostaderos, a lo que recientemente se ha sumado el incremento en visita pública (Comité Regional de la Sierra de Quila A.C. 2013).

A pesar del deterioro que muestran algunos tipos de vegetación dentro del área, la presencia de un buen número de especies de carnívoros nos da una idea de la resiliencia del ecosistema, en el que incluso podemos encontrar especies que son prioritarias para establecer programas de conservación, ya que se tienen el registro de dos especies de felinos con categoría en peligro de extinción (P) según la NOM-ECOL-059-2010 (DOF 2010). Estas especies fueron registradas en cañadas, las cuales aún conservan vegetación densa, necesaria para ser utilizada como refugio.

Figura 2. Patrones de

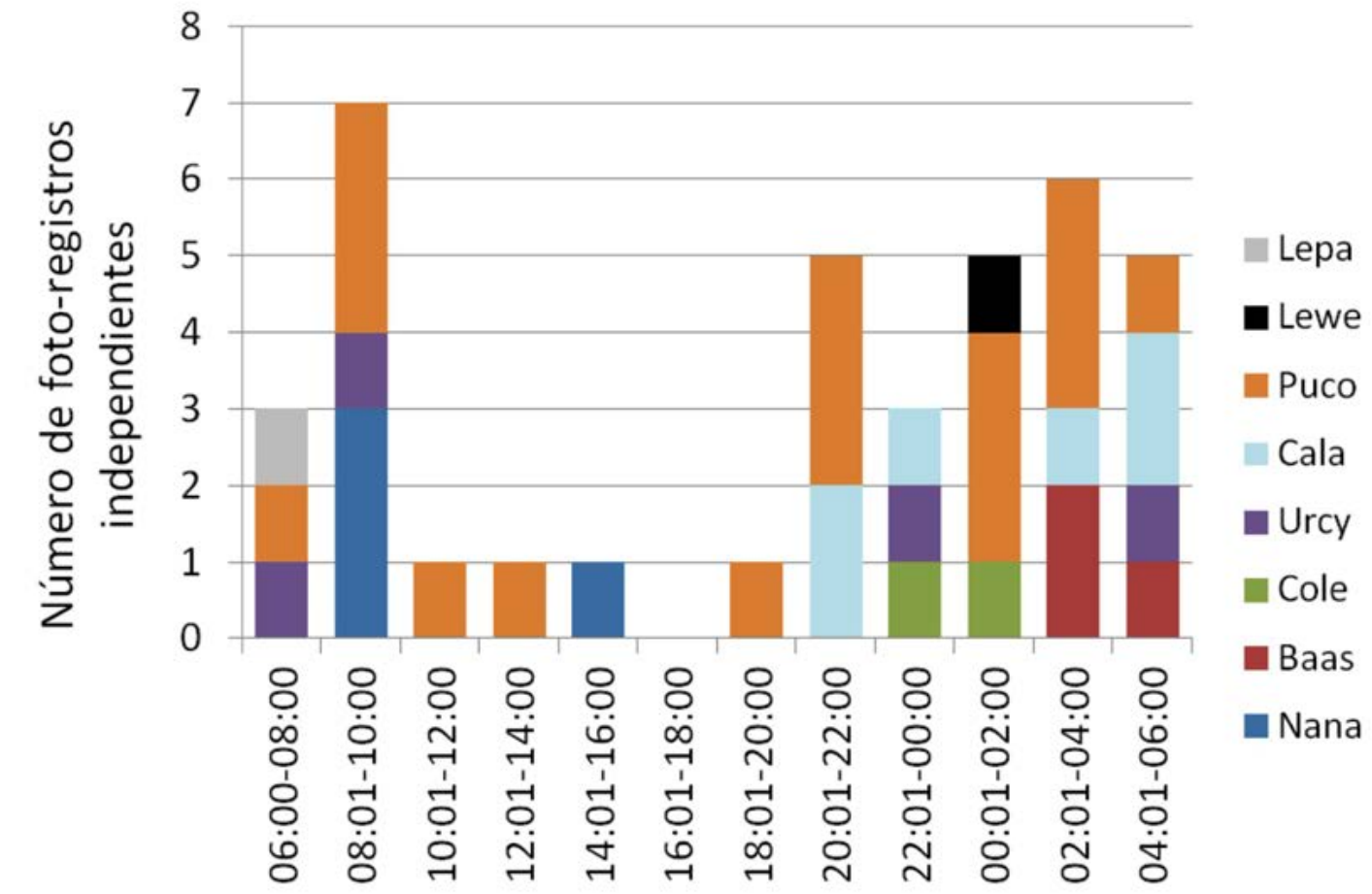
de Sierra de Quila, con base en los registros de cámaras trampa. Acrónimos: Lepa: Leopardus pardalis, Lewe: Leopardus weidii, Puco: Puma concolor, Cala: Canis latrans, Urcy: Urocyon cinereoargenteus, Cole: Conepatus leuconotus, Baas: Bassariscus astutus y Nana: Nasua narica.

\section{Horario}

En trabajos futuros en la Sierra de Quila, el esfuerzo de muestreo debe dirigirse a los sitios menos representados en este trabajo o donde no fue posible muestrear; tal es el caso de la selva baja y partes bajas de los ríos, respectivamente. De acuerdo a testimonios de algunos trabajadores y pobladores del área, reportan avistamientos de nutrias, mapaches, linces y leoncillos en la parte baja del ANP. El registro de estas especies podría complementar el listado de carnívoros para el APFFSQ; incluso podrían existir registros de jaguar, aunque no ha sido reportado para esta área, debido a que no se puede descartar que utilice la Sierra de Quila como corredor de tránsito entre las montañas y la costa de Jalisco.

Este proyecto fue apoyado con recursos del Comité Regional de Protección, Promoción y Fomento de los Recursos Naturales de la Sierra de Quila, A. C., a través de la Dirección 
del APFFSQ, así como de la Universidad de Guadalajara. Agradecemos a tres árbitros anónimos que nos ayudaron a mejorar este manuscrito.

Agraz, G. de A. G. 1950. Esbozos históricos de Tecolotlán (Jalisco). Talleres Linotipográficos El Estudiante S. A. Guadalajara, México.

Aranda, M. 2000. Huellas y otros rastros de los mamíferos grandes y medianos de México. Instituto de Ecología-Comisión Nacional para el Conocimiento y Uso de la Biodiversidad. Ciudad de México, México.

Aranda, M. 2012. Manual para el rastreo de mamíferos silvestres de México. Comisión Nacional para el Conocimiento y Uso de la Biodiversidad. Ciudad de México, México

Bond, W. 2010. Consumer control by megafauna and fire. Pp. 275-285 en Trophic cascades. Predators, prey and the changing dynamics of nature (J. Terborgh y J. Estes, eds.). Island Press. Washington, EE.UU.

Caro, T. M. y G. O'Doherty. 1999. On the use of surrogate species in conservation biology. Conservation Biology 13:805-814.

CEA (Comisión Estatal de Agua - Jalisco). 2011. Conjunto de datos geográficos de la Carta Hidrológica de Aguas Superficiales, 1:250,000. CEA Jalisco, SIAEJ (Sistema de Información del Agua). Guadalajara, México.

Comité Regional de la Sierra de Quila A.C. 2013. Programa de Manejo Área de Protección de Flora y Fauna Sierra de Quila. Documento en proceso de consulta pública. Fecha de publicación: 02/10/2012. Fecha de consulta: 12/08/2013. Liga para consulta: http://sierradequila.org/sdq/index.php?option=com_docman\&task=cat_ view\&gid=22\&ltemid $=30$

Day, G. I., S. D. Schemnitz, y R. D. Taber. 1987. Captura y marcación de animales silvestres. Pp. 63-94 en Manual de técnicas de gestión de vida silvestre ( $R$. Rodríguez Tarres, ed.). The Wildlife Society. Bethseda, EE.UU.

DOF (Diario Oficial de la Federación). 2010. Norma Oficial Mexicana (NOM-059SEMARNAT-2010, Protección ambiental-Especies nativas de México de flora y fauna silvestres-Categorías de Riesgo y especificaciones para su inclusión, exclusión o cambio y lista de especies de riesgo. Secretaría del Medio Ambiente y Recursos Naturales (SEMARNAT). 30 de diciembre de 2010. Ciudad de México, México

Escalante Pliego, P., A. G. Navarro Sigüenza, y A. T. Peterson. 1998. Un análisis geográfico, ecológico e histórico de la diversidad de aves terrestres de México. Pp. 279304 en Diversidad biológica de México. Orígenes y distribución (Ramamoorthy, T. P., R. Bye, A. Lot, y J. Fa, comps.). Instituto de Biología, Universidad Nacional Autónoma de México. Ciudad de México, México.

FA, J. E., Y L. M. Morales. 1998. Patrones de diversidad de mamíferos de México. Pp. 315352 en Diversidad biológica de México. Orígenes y distribución (Ramamoorthy, T. P., R. Bye, A. Lot, y J. Fa, comps.). Instituto de Biología, Universidad Nacional Autónoma de México. Ciudad de México, México.

Feinsinger, P. 2001. Designing field studies for biodiversity conservation. The Nature Conservancy-Island Press. Washington, EE.UU. 
Flores Villela, O., y P. Geréz. 1994. Biodiversidad conservación en México: Vertebrados, vegetación y uso del suelo. Comisión Nacional para el Uso y Conocimiento de la Biodiversidad, y Universidad Nacional Autónoma de México. Ciudad de México, México

Flores Villela, O. 1998. Herpetofauna de México: Distribución y endemismo. Pp. 251 278 en Diversidad biológica de México. Orígenes y distribución (Ramamoorthy, T. P., R. Bye, A. Lot, y J. Fa, comps.). Instituto de Biología, Universidad Nacional Autónoma de México. Ciudad de México, México

García, A., y G. Ceballos. 1994. Guía de campo de los reptiles y anfibios de la costa de Jalisco, México. Fundación Ecológica Cuixmala, A. C. e Instituto de Biología, UNAM. Ciudad de México, México.

García, E. 1988. Modificaciones al sistema de clasificación climática de Köppen (para adaptarlo a las condiciones de la Republica Mexicana). Instituto de Geografía, Universidad Nacional Autónoma de México. Ciudad de México, México.

Godínez, E. G., N. González-Ruiz, y J. Ramírez-Pulido. 2011. Actualización de la lista de los mamíferos de Jalisco, México: implicaciones en los cambios taxonómicos. Therya 2:7-35.

Guerrero, N. J. J., y M. Cházaro. 1995. Datos generales sobre la Sierra de Quila, Jalisco. Pp. 70-73 en Antología botánica del Estado de Jalisco, México (Chazáro B., M., M. E. Lomelí, R. R. Acevedo, y R. S. Ellerbracke, comps.). Departamento de Geografía y Ordenación Territorial, Universidad de Guadalajara. Guadalajara, México.

Iñıguez Dávalos, L. I. ,Y E. Santana-C. 1993. Patrones de distribución y riqueza de especies de los mamíferos del occidente de México. Pp. 65-86 en Avances en el estudio de los mamíferos de México (Medellín, R., y G. Ceballos, eds.). Asociación Mexicana de Mastozoología, A.C. Publicaciones especiales, Vol. 1. Ciudad de México, México.

Iñıguez Dávalos, L. I., y E. Santana-C. 2004. Análisis mastofaunístico del Estado de Jalisco. Pp. 251-258 en Contribuciones mastozoológicas en homenaje a Bernardo Villa (Sánchez-Cordero, V., y R. Medellín, eds.). Instituto de Biología e Instituto de Ecología, Universidad Nacional Autónoma de México. Ciudad de México, México.

Jardel, E. J., E. Santana C., y S. Graf-Montero. 1996. The Sierra de Manantlán Biosphere Reserve: Conservation and regional sustainable development. Parks 6:14- 22.

Monroy-Vilchis, O., M. M. Zarco-González, C. Rodríguez-Soto, L. Soria-Díaz, y V. UriosMouiner. 2011. Fototrampeo de mamíferos en la Sierra Nanchititla, México: abundancia relativa y patrón de actividad. Revista de Biología Tropical 59:373383.

Ramamoorty, T. P., R. Bye, A. Lott, y J. Fa (comps.). 1998. Diversidad biológica de México. Instituto de Biología, Universidad Nacional Autónoma de México. Ciudad de México, México.

SEMADES. 2010. Áreas Naturales Protegidas de Jalisco. Secretaría de Medio Ambiente para el Desarrollo Sustentable (SEMADES). Tomado de http://www.semades. jalisco.gob.mx. Fecha de consulta: 7 de enero de 2011. 
Spowart, R. A., y F. B. Samson. 1986. Carnivores. Pp. 475-496 en Inventory and monitoring of wildlife habitat (Cooperrider, A. Y., R. J. Boyd, y H. R. Stuart, eds.). Bureau of Land Management, U.S. Dept. of the Interior. Denver, EE.UU.

Tattersall, I. 2006. The concept of cathemerality: History and definition. Folia Primatologica 77:7-14.

Terborgh, J., R. D. Holt, y J. A. Estes. 2010. Trophic cascades: What they are, how they work, and why they matter. Pp. 1-18 en Trophic cascades. Predators, prey and the changing dynamics of nature (J. Terborgh y J. Estes, eds.). Island Press. Washington, EE.UU.

Van Schaik, P., y M. Griffiths. 1996. Activity periods of Indonesian rain forest mammals. Biotropica 28:105-112.

Villavicencio G., R., P. Bauche, A. Gallegos, A. L. Santiago P., y F. M. Huerta M. 2005. Caracterización estructural y diversidad de comunidades arbóreas de la Sierra de Quila. Ibugana 13:67-76.

YASUDA, M. 2004. Monitoring diversity and abundance of mammals with camera traps: a case study on Mount Tsukuba, central Japan. Mammal Study 29:37-46.

Sometido: 13 de septiembre de 2013

Revisado: 21 de noviembre de 2013

Aceptado: 7 de agosto de 2014

Editor asociado: Jorge Servin

Diseño gráfico editorial: Gerardo Hernández 